\title{
High Precision Test of the Pauli Exclusion Principle for Electrons
}

Kristian Piscicchia ${ }^{1,2, *}$, Aidin Amirkhani ${ }^{3}$, Sergio Bartalucci ${ }^{2}$, Sergio Bertolucci ${ }^{4}$,
Massimiliano Bazzi ${ }^{2}$, Mario Bragadireanu ${ }^{2,5}$, Michael Cargnelli ${ }^{6}$, Alberto Clozza ${ }^{2}$,
Catalina Curceanu $1,2,5$, Raffaele Del Grande ${ }^{2}$, Luca De Paolis ${ }^{2}$ Jean-Pierre Egger ${ }^{7}$ Catalna Curceanu 1,2, Raffaele Del Grande ${ }^{2}$, Luca De Paolis ', Jean-Pierre Egger ${ }^{7}$, Carlo Fiorini ${ }^{3}$, Carlo Guaraldo ${ }^{2}(0)$, Mihail Iliescu ${ }^{2}$, Matthias Laubenstein ${ }^{8}$, Johann Marton ${ }^{6}$, Marco Miliucci ${ }^{2}{ }^{-1}$, Edoardo Milotti ${ }^{9}$, Andreas Pichler ${ }^{6}$, Dorel Pietreanu ${ }^{2,5}$, Alessandro Scordo ${ }^{2}$, Hexi Shi ${ }^{10}$, Diana Laura Sirghi ${ }^{2,5}$, Florin Sirghi ${ }^{2,5}$, Laura Sperandio ${ }^{2}$, Oton Vazquez Doce ${ }^{11}$ and Johann Zmeskal ${ }^{6}$

1 Centro Fermi-Museo Storico della Fisica e Centro Studi e Ricerche “Enrico Fermi", 00184 Rome, Italy;

Catalina.Curceanu@lnf.infn.it

2 Laboratori Nazionali di Frascati, INFN, 00044 Frascati, Italy; Sergio.bartalucci@lnf.infn.it (S.B.); massimiliano.bazzi@lnf.infn.it (M.B.); bragadireanu.mario@lnf.infn.it (M.B.); alberto.clozza@lnf.infn.it (A.C.); raffaele.delgrande@lnf.infn.it (R.D.G.); Luca.DePaolis@lnf.infn.it (L.D.P.); guaraldo@lnf.infn.it (C.G.); mihai.iliescu@lnf.infn.it (M.I.); marco.miliucci@lnf.infn.it (M.M.); dorel.pietreanu@lnf.infn.it (D.P.); scordo@lnf.infn.it (A.S.); sirghi@lnf.infn.it (D.L.S.); fsirghi@lnf.infn.it (F.S.); Laura.Sperandio@lnf.infn.it (L.S.)

3 Politecnico di Milano, Dipartimento di Elettronica, Informazione e Bioingegneria and INFN Sezione di Milano, 20133 Milano, Italy; aidin.amirkhani@polimi.it (A.A.); carlo.fiorini@polimi.it (C.F.)

4 Dipartimento di Fisica e Astronomia, Università di Bologna, 40126 Bologna, Italy; Sergio.Bertolucci@Inf.infn.it

5 IFIN-HH, Institutul National pentru Fizica si Inginerie Nucleara Horia Hulubei, Magurele 077125, Romania

6 Stefan-Meyer-Institute for Subatomic Physics, Austrian Academy of Science, 1090 Vienna, Austria; michael.cargnelli@oeaw.ac.at (M.C.); johann.marton@oeaw.ac.at (J.M.); Andreas.Pichler@oeaw.ac.at (A.P.); johann.zmeskal@oeaw.ac.at (J.Z.)

7 Institut de Physique, Université de Neuchâtel, CH-2000 Neuchâtel, Switzerland; jean-pierre.egger@net2000.ch

8 Laboratori Nazionali del Gran Sasso, INFN, 67100 Assergi, Italy; matthias.laubenstein@lngs.infn.it

9 Dipartimento di Fisica, Università di Trieste and INFN-Sezione di Trieste, 34127 Trieste, Italy; milotti@ts.infn.it

10 Institut für Hochenergiephysik der Österreichischen Akademie der Wissenschaften, 1050 Vienna, Austria; shihexi@gmail.com

11 Excellence Cluster Universe, Technische Universität München, D-85748 Garching, Germany; otonvazquezdoce@gmail.com

* Correspondence: kristian.piscicchia@lnf.infn.it

Received: 6 March 2019; Accepted: 23 April 2019; Published: 2 May 2019

Abstract: The VIP-2 experiment aims to perform high precision tests of the Pauli Exclusion Principle for electrons. The method consists in circulating a continuous current in a copper strip, searching for the $X$ radiation emission due to a prohibited transition (from the $2 p$ level to the $1 \mathrm{~s}$ level of copper when this is already occupied by two electrons). VIP already set the best limit on the PEP violation probability for electrons $\frac{1}{2} \beta^{2}<4.7 \times 10^{-29}$, the goal of the upgraded VIP-2 (VIolation of the Pauli Exclusion Principle-2) experiment is to improve this result of two orders of magnitude at least. The experimental apparatus and the results of the analysis of a first set of collected data will be presented.

Keywords: Pauli exclusion principle; quantum foundations; X-ray spectroscopy; underground experiment 


\section{Introduction}

The VIP collaboration is performing high precision tests of the Pauli Exclusion Principle (PEP) for electrons, in the extremely low cosmic background environment of the Underground Gran Sasso Laboratories (LNGS) of INFN (Italy). According to the PEP a system can not hold two (or more) fermions with all quantum numbers identical. PEP stands as one of the fundamental and most solid cornerstones of modern physics, its validity explaining a plenty of phenomena such as the structure of atoms. The PEP was originally formulated for the electrons (see Ref. [1]) and was later extended to all the fermions by the spin-statistics theorem, which can only be demonstrated within Quantum Field Theory (QFT). According to the spin-statistics connection the quantum states of identical particles are necessarily either symmetric (for bosons) or antisymmetric (for fermions) with respect to their permutation. Extensions of the QFT admit, however, spin-statistics violations, hence experimental evidence of even a tiny violation of the PEP would be an indication of physics beyond the Standard Model.

VIP (see Refs. [2-4]) greatly improved an experimental technique conceived by Ramberg and Snow (see Ref. [5]) which consists in circulating a DC current in a copper conductor and search for the X-rays signature of PEP-violating $K_{\alpha}$ transitions $(2 p \rightarrow 1 s$ in $\mathrm{Cu}$ when the 1 s level is already occupied by two electrons). As a consequence of the shielding effect of the two electrons in the ground state, the $K_{\alpha}$ violating transition is shifted of about $300 \mathrm{eV}$ with respect to the standard line and is then distinguishable in precision spectroscopic measurements. Such experimental procedure aims to evidence an anomalous behaviour of the newly injected electrons which never had before the possibility to perform the searched violating $K_{\alpha}$ transition in the target $\mathrm{Cu}$ atoms. In this sense VIP strictly fulfills the Messiah-Greenberg superselection rule [6] which excludes transitions between different symmetry states in a given system. Considering open systems is then a crucial feature in order to consistently test a violation of the PEP, whose probability is usually quantified by means of the $\beta^{2} / 2$ parameter $[7,8]$.

In what follows the upgraded VIP-2 experimental apparatus (see Refs. $[9,10])$ will be presented, and the analysis of a first set of data (collected in 2016) will be described. As will be shown VIP-2 already improved the upper limit imposed by VIP on $\beta^{2} / 2$ (after three years of data taking), which represents the best limit ever on the PEP violation probability for electrons. The final goal of VIP-2 (which is presently acquiring data) is to either further improve the limit of two orders of magnitude, or to measure a signal of PEP violation.

\section{The VIP-2 Experimental Apparatus}

VIP-2 is the upgraded version of the VIP experiment and aims to improve the result obtained by VIP of two orders of magnitude at least. VIP set the best limit on the PEP violation probability for electrons $\frac{1}{2} \beta^{2}<4.7 \times 10^{-29}$ [2] exploiting the experimental technique which was pioneered by Ramberg and Snow. The VIP experimental setup made use of Charge Coupled Devices (CCDs) as the X-ray detectors; CCDs were characterised by a Full Width at Half Maximum (FWHM) of $320 \mathrm{eV}$ at $8 \mathrm{keV}$, corresponding to the definition of the Region Of Interest (ROI) where the signature of anomalous $\mathrm{X}$-ray transitions is searched for. Moreover, VIP was operated in the extremely low cosmic background environment of the Underground Gran Sasso Laboratories (LNGS) of INFN.

The goal of VIP-2 will be achieved by implementing many improvements in the experimental apparatus. The core components of the VIP-2 setup are illustrated in Figure 1. The new layout of the copper target consists of two strips of copper (with a thickness of $50 \mu \mathrm{m}$, and a surface of $9 \mathrm{~cm} \times 2 \mathrm{~cm}$ ) the new geometry results in a higher acceptance for the $X$-ray detection. The heat due to the dissipation in copper would lead to a significant temperature rise in the strip. In order to avoid this effect a cooling pad (cooled down by a closed chiller circuit) is placed in between the two strips. This also allows to enhance the DC current circulating on the strips to $100 \mathrm{~A}$ (instead of the $40 \mathrm{~A}$ in VIP) thus increasing the candidate event pool for the anomalous X-rays. The CCDs were replaced by Silicon Drift Detectors (SDDs) as X-ray detectors, with a better energy resolution (190 eV FWHM at $8 \mathrm{keV})$. The data presented 
in this work were acquired by means of two arrays of $1 \times 3$ SDDs surrounding the copper target, each array with $3 \mathrm{~cm}^{2}$ of effective surface. The SDDs were cooled down to $-170{ }^{\circ} \mathrm{C}$ with circulating liquid argon in a closed cooling line. With a current of $100 \mathrm{~A}$ circulating in the strips, their temperature rises up by about $20^{\circ} \mathrm{C}$, inducing a temperature rise at the SDDs of about $1 \mathrm{~K}$, which does not significantly alters the SDDs performances.

The timing capability of the SDDs also enables to introduce an active shielding system. This veto system is made of 32 plastic scintillator bars $(250 \mathrm{~mm} \times 38 \mathrm{~mm} \times 40 \mathrm{~mm}$ bar $)$ surrounding the SDDs and serves to remove the background originating from the high energy charged particles that are not shielded by the rocks of the Gran Sasso mountains. The light output of each scintillator is read out by two silicon photomultipliers (SiPMs) coupled to each end of the bars.

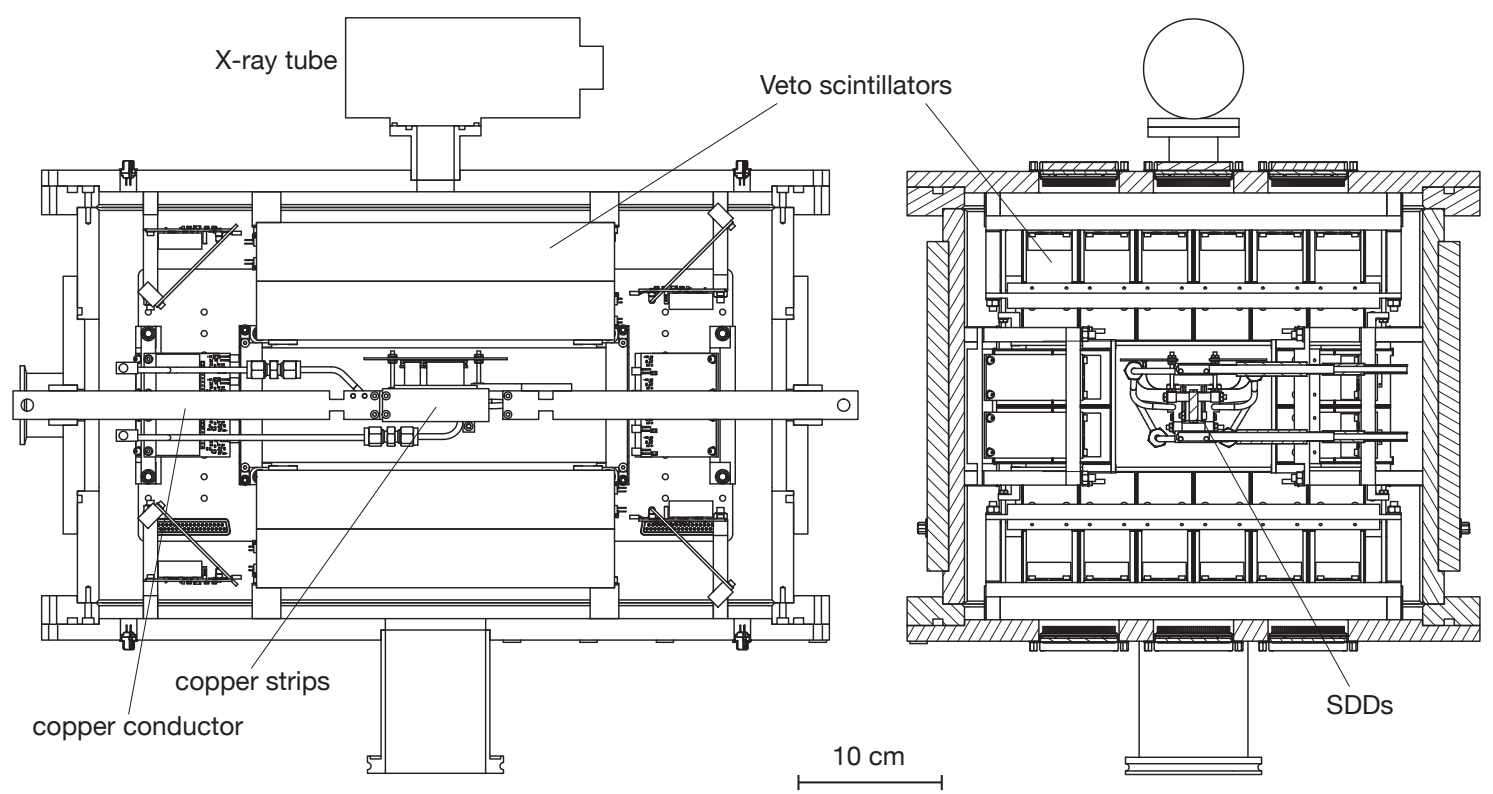

Figure 1. The side views of the design of the core components of the VIP-2 setup, including the SDDs as the $\mathrm{X}$-ray detector, the scintillators as active shielding with silicon photomultiplier readout.

All the detectors and the front end preamplifier electronics are mounted inside the vacuum chamber which is kept at $10^{-5}$ mbar during operation.

In order to perform quick energy calibration and SDDs resolution measurements an X-ray tube on top of the setup irradiates Zirconium and Titanium foils, to produce fluorescence reference lines. A Kapton window in the vacuum chamber and an opening solid angle in the upper scintillator bars, allow to collect in one hour enough statistics for the SDDs performance monitoring. A secondary energy calibration method of the SDDs is performed by means of a weakly radioactive Fe-55 source, with a $25 \mu \mathrm{m}$ thick Titanium foil attached on top, mounted together inside an aluminum holder. The six SDDs have an overall $2 \mathrm{~Hz}$ trigger rate, accumulating events of fluorescence X-rays from titanium and manganese to calibrate the digitized channel into energy scale.

The VIP-2 experimental apparatus was transported and mounted in the LNGS at the end of 2015. Following a period of tuning and optimization a first campaign of data taking started from October 2016 with the complete detector system (except the passive shielding). A total amount of 34 days of data with a 100 A DC current and 28 days without current were collected until the end of the year 2016. In the next section, the analysis of this data set and the obtained result are shown.

The VIP-2 setup was further upgraded during 2018: new copper targets were realised, the SDDs arrays were replaced with two arrays $2 \times 8$ for a total of 32 SDDs and the passive shielding was mounted. The passive shielding, which is made of two layers of lead and copper blocks, will kill most of the background due to environmental gamma radiation. The final configuration of the VIP-2 setup, which is presently taking data, is shown in Figure 2. The energy calibrated spectra corresponding to an 
equal data collection period of 39 days during 2018, with and without current, are shown in Figure 3 left and right respectively. The data analysis, performed with a similar procedure to that described in Section 3, is presently ongoing on this data set.

More details on the VIP-2 experimental apparatus, the trigger logic, data acquisition and slow control can be found in Ref. [9].

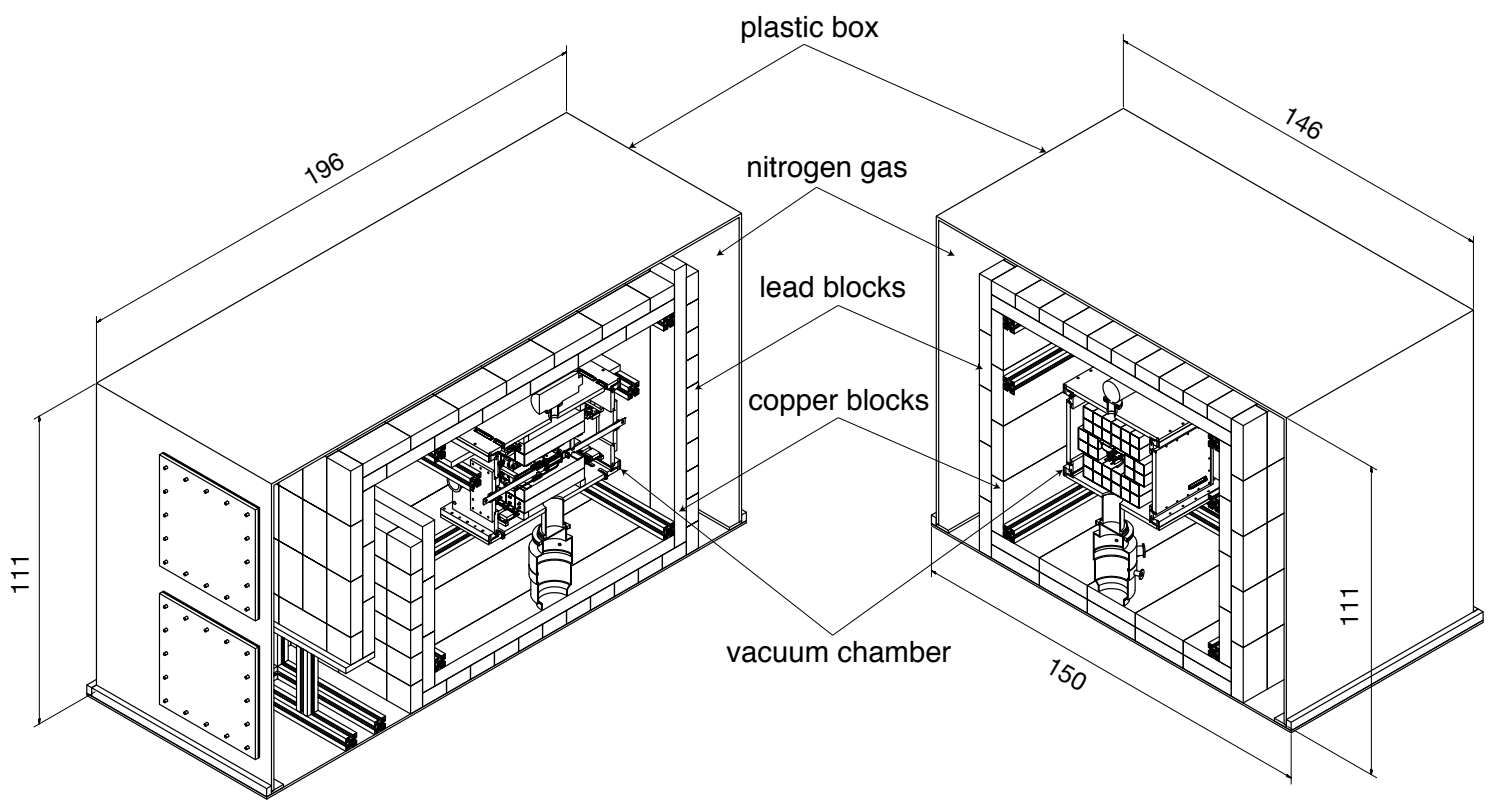

Figure 2. Perspective views of the VIP-2 apparatus with passive shielding, with the dimensions in $\mathrm{cm}$. Nitrogen gas with a slight over pressure with respect to the external air will be circulated inside a plastic box in order to reduce the radon contamination.
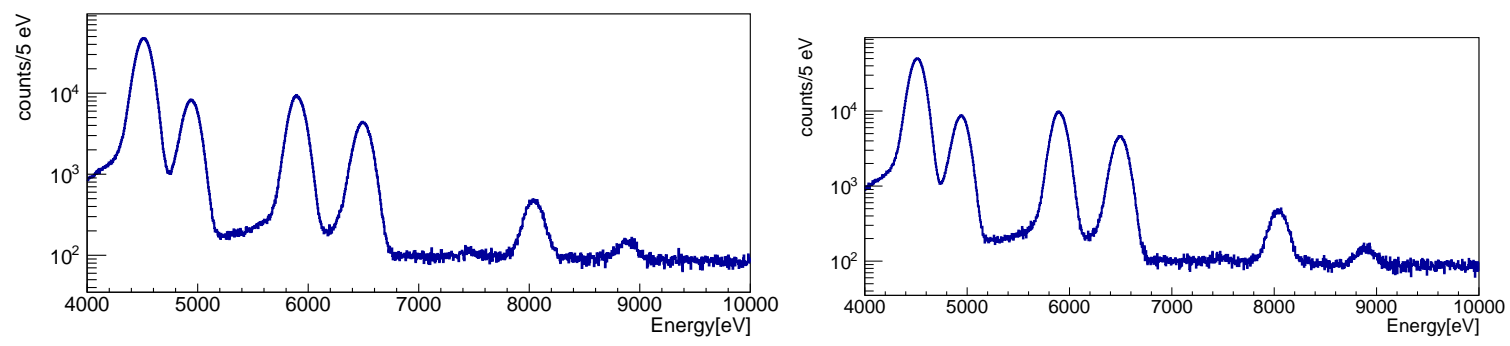

Figure 3. Energy calibrated spectra corresponding to 39 days of data taking without current (left) and 39 days with 100 A DC current (right) collected during 2018.

\section{Data Analysis}

In order to put in evidence an eventual signal of PEP violating $\mathrm{K}_{\alpha}$ transitions a simultaneous fit was performed of the two spectra collected with and without current; the spectra and the obtained fit result are shown in Figure 4. The fit was performed by minimising a global Chi-square function, which is obtained as the product of the likelihoods corresponding to the two spectra, assuming the measurement errors to be distributed according to Gaussians. The fit proceeds in two steps: as first (see Figure 4a) a wide energy range is used (from $3.5 \mathrm{keV}$ to $11 \mathrm{keV}$ ) in order to exploit the high statistics titanium and manganese lines to determine the Fano Factor and the Constant Noise (an energy independent contribution to the energy resolution). The parameters obtained from this pre-fit are then used as an input for the second fit in the range from $7 \mathrm{keV}$ to $11 \mathrm{keV}$ (see Figure $4 \mathrm{~b}$ top), from which the shape of the continuous background near the interesting transition is better determined. The fit parameters accounting for the detector energy resolution, the shape of the continuous background, the shape of the fluorescence peaks, are common for the spectra with and without current. The parameters 
representing the intensities of the fluorescence peaks and of the continuous background are separately defined. For the current on spectrum an additional Gaussian component was introduced representing the eventual PEP violating $\mathrm{K}_{\alpha}$ transition line, the centre of the line was set at $7746.73 \mathrm{eV}$ (see Refs. [9]). In Figure $4 \mathrm{~b}$ bottom the residuals from the second fit are shown for the two spectra. The Chi-square minimisation was performed using the MINUIT package of the CERN ROOT software framework [11]. From the fit the number of candidate PEP violating events, contributing to the $K_{\alpha}$ violating transitions, is obtained, together with the corresponding statistical error:

$$
N_{X}=54 \pm 67 \text { (statistical). }
$$

By analogy with the original limit estimated by Ramberg and Snow in Ref. [5] $N_{X}$ can be related to the PEP violation probability $\frac{1}{2} \beta^{2}$ as follows:

$$
N_{X} \geq \frac{1}{2} \beta^{2} \cdot N_{\text {new }} \cdot \frac{1}{10} \cdot N_{\text {int }} \cdot \epsilon
$$

In Equation (2) $N_{\text {new }}=(1 / e) \int_{\Delta t} I(t) d t$ is the number of current electrons injected in the copper target over the acquisition time period (with current) $\Delta t$, the factor $1 / 10$ accounts for the capture probability (per electron-atom scattering) into the $2 p$ state (see Ref. [12]), $N_{\text {int }}=D / \mu$ is the minimum number of electron-atom scatterings, where $D$ is the effective length of the copper strip and $\mu$ the scattering length for conduction electrons in the copper strip, to conclude $\epsilon=1.8 \%$ is the detection efficiency factor, obtained by means of a Monte Carlo (MC) simulation (as described in [9]). By substituting $\mu=3.9 \times 10^{-6} \mathrm{~cm}, e=1.602 \times 10^{-19} \mathrm{C}, I=100 \mathrm{~A}$, and the effective length of the copper strip $D=7.1 \mathrm{~cm}$ (the same used in the $\mathrm{MC}$ simulation), using the three sigma upper bound of $3 \cdot \Delta N_{X}=201$ to give a $99.7 \%$ C.L., the following upper limit is obtained for the PEP violation probability:

$$
\frac{\beta^{2}}{2} \leq 3.4 \cdot 10^{-29}
$$

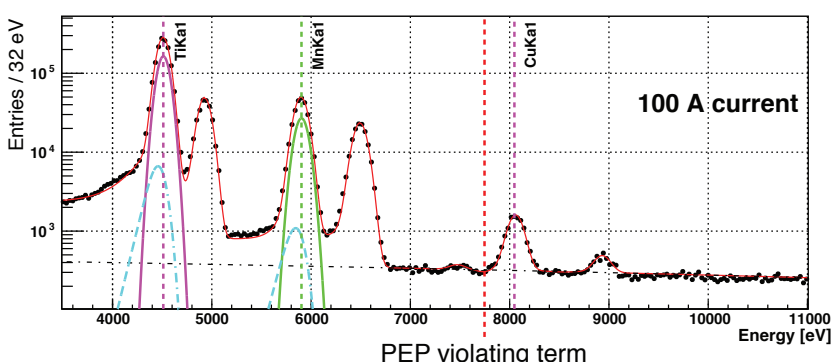

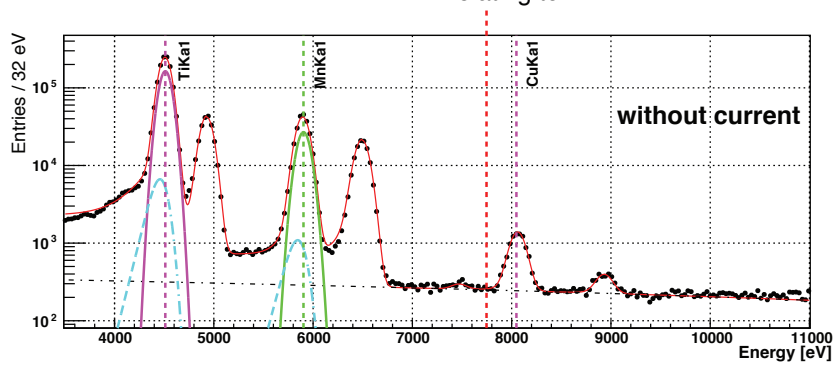

(a)
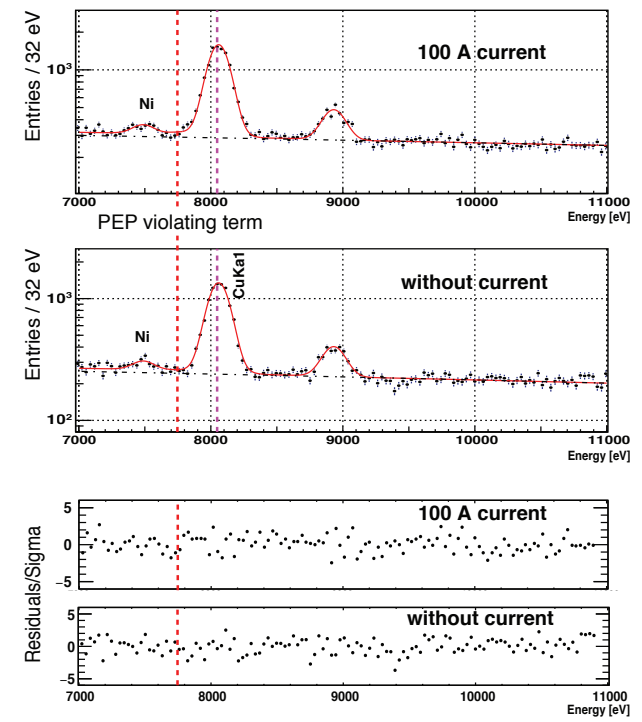

(b)

Figure 4. A global chi-square function was used to fit simultaneously the spectra with and without 100 A current applied to the copper conductor. The energy position for the expected PEP violating events is about $300 \mathrm{eV}$ below the normal copper $K_{\alpha 1}$ transition. The Gaussian function and the tail part of the $K_{\alpha 1}$ components and the continuous background from the fit result are also plotted. (a): the fit to the wide energy range from $3.5 \mathrm{keV}$ to $11 \mathrm{keV}$; (b): the fit and its residual for the $7 \mathrm{keV}$ to $11 \mathrm{keV}$ range where there is no background coming from the calibration source. See the main text for details. 


\section{Discussion and Perspectives}

In analogy with the analysis performed by Ramberg and Snow, the limit obtained in Equation (3) assumes a very simple straight path of the electrons across the $\mathrm{Cu}$ target strip. As a consequence, the scattering length is used in order to estimate the number of electron capture processes. In Ref. [13] it is argued that scatterings are not actually related to the atoms themselves, but depend on impurities, lattice imperfections and on phonons. For this reason the mean time between close electron-atom encounters is instead evaluated in Ref. [13], which is found to be $3.5 \cdot 10^{-17} \mathrm{~s}$ (instead of the much longer average scattering time $2.5 \times 10^{-14}$ ). Considered the traversal time of the copper target (which is estimated in [13] to amount to $10 \mathrm{~s}$ for the setup described in Section 2) an improved limit is obtained on the PEP violation probability: $\frac{\beta^{2}}{2} \leq 2.6 \cdot 10^{-40}$.

The analysis presented in Ref. [13] for the complex random walk which electron undergo in crossing the copper target material is mostly classical. We are presently working to extend the calculation to the quantum domain.

Author Contributions: Conceptualization, S.B. (Sergio Bertolucci), M.B. (Massimiliano Bazzi), A.C., C.C., J.-P.E., C.G., M.I., M.L., J.M., E.M., A.P., D.P., J.Z.; software, K.P., M.C., R.D.G., L.S., D.P., M.S., O.V.D., D.S., H.S.; formal analysis, K.P., H.S., A.P., D.P., R.D.G., M.M., L.D.P.; data curation A.A., C.F., M.C., S.B. (Sergio Bartalucci), M.B. (Mario Bragadireanu), A.C., C.C., L.D.P., M.I., M.L., J.M., M.M., A.P., D.P., A.S., D.L.S., F.S., H.S., J.Z.; writing and editing, K.P., C.C., E.M.; supervision, C.C.

Funding: "This research was partially funded by Centro Fermi-Museo Storico della Fisica e Centro Studi e Ricerche "Enrico Fermi" (Open Problems in Quantum Mechanics project) and by the Austrian Science Foundation (FWF) with the grants P25529-N20, project P 30635-N36 and W1252-N27".

Acknowledgments: We thank Herbert Schneider, Leopold Stohwasser, and Doris Pristauz-Telsnigg from Stefan-Meyer-Institut for their fundamental contribution in designing and building the VIP2 setup. We acknowledge the very important assistance of the INFN-LNGS laboratory. We acknowledge the support of the Centro Fermi-Museo Storico della Fisica e Centro Studi e Ricerche "Enrico Fermi" (Open Problems in Quantum Mechanics project), the support from the EU COST Action CA 15220 and of the EU FET project TEQ (grant agreement 766900) is gratefully acknowledged. We thank the Austrian Science Foundation (FWF) which supports the VIP2 project with the grants P25529-N20, project P 30635-N36 and W1252-N27 (doctoral college particles and interactions). Furthermore, these studies were made possible through the support of a grant from the Foundational Questions Institute, FOXi (and a grant from the John Templeton Foundation (ID 58158). The opinions expressed in this publication are those of the authors and do not necessarily respect the views of the John Templeton Foundation.

Conflicts of Interest: The authors declare no conflict of interest.

\section{References}

1. Pauli, W. Über den Zusammenhang des Abschlusses der Elektronengruppen im Atom mit der Komplexstruktur der Spektren. Z. Phys. 1925, 31, 765. [CrossRef]

2. Petrascu, C.C.; Bartalucci, S.; Bertolucci, S.; Bragadireanu, M.; Cargnelli, M.; Di Matteo, S.; Egger, J.-P.; Guaraldo, C.; Iliescu, M.; Ishiwatari, T. Experimental tests of quantum mechanics-Pauli exclusion principle violation (the VIP experiment) and future perspective. J. Phys. Conf. Ser. 2011, 306, 012036. [CrossRef]

3. Bartalucci, S.; Bertolucci, S.; Bragadireanu, M.; Cargnelli, M.; Curceanu, C.; Di Matteo, S.; Egger, J.-P.; Guaraldo, C.; Iliescu, M.; Ishiwatari, T. The VIP experimental limit on the Pauli exclusion principle violation by electrons. Found. Phys. 2010, 40, 765-775. [CrossRef]

4. Sperandio, L. New Experimental Limit on the Pauli Exclusion Principle Violation by Electrons From the VIP Experiment. Ph.D. Thesis, Tor Vergata University, Rome, Italy, 2008.

5. Ramberg, E.; Snow, G.A. Experimental limit on a small violation of the Pauli principle. Phys. Lett. B. 1990, 238, 438-441. [CrossRef]

6. Messiah, A.; Greenberg, O. Symmetrization postulate and its experimental foundation. Phys. Rev. 1964, 136, B248. [CrossRef]

7. Ignatiev, A.Y.; Kuzmin, V. Quarks '86: Proceedings of the Seminar, Tbilisi, USSR, 15-17 April 1986; Tavkhelidze, A.N., Matveev, V.A., Pivovarov, A.A., Tkachev, I.I., Eds.; VNU Science Press BV: Utrecht, The Netherlands, 1987; pp. 263-268.

8. Ignatiev, A.Y. X rays test the Pauli exclusion principle. Radiat. Phys. Chem. 2006, 75, 2090-2096. [CrossRef] 
9. Shi, H.; Milotti, E.; Bartalucci, S.; Bazzi, M.; Bertolucci, S.; Bragadireanu, A.; Cargnelli, M.; Clozza, A.; De Paolis, L.; Di Matteo, S.; et al. Experimental search for the violation of Pauli exclusion principle. Eur. Phys. J. C 2018, 78, 319. [CrossRef] [PubMed]

10. Curceanu, C.; Shi, H.; Bartalucci, S.; Bertolucci, S.; Bazzi, M.; Berucci, C.; Bragadireanu, M.; Cargnelli, M.; Clozza, A.; De Paolis, L.; et al. Test of the Pauli Exclusion Principle in the VIP-2 underground experiment. Entropy 2017, 19, 300. [CrossRef]

11. Brun, R.; Rademakers, F. ROOT-An object oriented data analysis framework. Nucl. Instrum. Methods Phys. Res. A 1997, 389, 81-86. [CrossRef]

12. Bartalucci, S.; Bertolucci, S.; Bragadireanu, M.; Cargnelli, M.; Catitti, M.; Curceanu, C.; Di Matteo, S.; Egger, J.-P.; Guaraldo, C.; Iliescu, M. New experimental limit on the Pauli exclusion principle violation by electrons. Phys. Lett. B 2006, 641, 18-22. [CrossRef]

13. Milotti, E.; Bartalucci, S.; Bertolucci, S.; Bazzi, M.; Bragadireanu, M.; Cargnelli, M.; Clozza, A.; Curceanu, C.; De Paolis, L.; Egger, J.-P.; et al. On the Importance of Electron Diffusion in a Bulk-Matter Test of the Pauli Exclusion Principle. Entropy 2018, 20, 515. [CrossRef]

(C) 2019 by the authors. Licensee MDPI, Basel, Switzerland. This article is an open access article distributed under the terms and conditions of the Creative Commons Attribution (CC BY) license (http://creativecommons.org/licenses/by/4.0/). 